The Journal of $\mathbf{N}_{\text {onlinear }} \mathbf{S}_{\text {ciences and }}$ Applications http://www.tjnsa.com

\title{
STABILITY OF AN ADDITIVE-QUADRATIC FUNCTIONAL EQUATION OF TWO VARIABLES IN F-SPACES
}

\author{
M. ESHAGHI GORDJI
}

ABSTRACT. In this paper, we achieve the Hyers-Ulam-Rassias stability of the following system of functional equations

$$
\left\{\begin{array}{l}
f\left(x_{1}+x_{2}, y\right)=f\left(x_{1}, y\right)+f\left(x_{2}, y\right) \\
f\left(x, y_{1}+y_{2}\right)+f\left(x, y_{1}-y_{2}\right)=2 f\left(x, y_{1}\right)+2 f\left(x, y_{2}\right)
\end{array}\right.
$$

in F-spaces.

\section{INTRODUCTION AND PRELIMINARIES}

The stability problem of functional equations originated from a question of Ulam [26] in 1940, concerning the stability of group homomorphisms. Let $\left(G_{1},.\right)$ be a group and let $\left(G_{2}, *\right)$ be a metric group with the metric $d(.,$.$) . Given \epsilon>0$, dose there exist a $\delta>0$, such that if a mapping $h: G_{1} \longrightarrow G_{2}$ satisfies the inequality $d(h(x . y), h(x) * h(y))<\delta$ for all $x, y \in G_{1}$, then there exists a homomorphism $H: G_{1} \longrightarrow G_{2}$ with $d(h(x), H(x))<\epsilon$ for all $x \in G_{1}$ ? In the other words, under what condition does there exist a homomorphism near an approximate homomorphism? The concept of stability for functional equation arises when we replace the functional equation by an inequality which acts as a perturbation of the equation. In 1941, D. H. Hyers [10] gave a first affirmative answer to the question of Ulam for Banach spaces. Let $f: E \longrightarrow E^{\prime}$ be a mapping between Banach spaces such that

$$
\|f(x+y)-f(x)-f(y)\| \leq \delta
$$

Date: Received: 2 March 2006; Revised: 15 August 15.

2000 Mathematics Subject Classification. Primary 39B82; Secondary 44B52.

Key words and phrases. Stability, functional equation. 
for all $x, y \in E$, and for some $\delta>0$. Then there exists a unique additive mapping $T: E \longrightarrow E^{\prime}$ such that

$$
\|f(x)-T(x)\| \leq \delta
$$

for all $x \in E$. Moreover if $f(t x)$ is continuous in t for each fixed $x \in E$, then $T$ is linear. In 1978, Th. M. Rassias [19]provided a generalization of Hyers' Theorem which allows the Cauchy difference to be unbounded (see also [20]).

In 1990, Th.M. Rassias [20] during the 27th International Symposium on Functional Equations asked the question whether such a theorem can also be proved for $p \geq 1$. In 1991, Gajda [8] gave an affirmative solution to this question for $p>1$ by following the same approach as in Rassias' paper [19]. It was proved by Gajda [8], as well as by Th.M. Rassias and Šemrl [22] that one cannot prove a Rassias type theorem when $p=1$. In 1994, P. Găvruta [9] provided a generalization of Rassias' theorem in which he replaced the bound $\varepsilon\left(\|x\|^{p}+\|y\|^{p}\right)$ in $([19])$ by a general control function $\varphi(x, y)$. The paper of Th.M. Rassias [19] has provided a lot of influence in the development of what we now call Hyers-Ulam-Rassias stability of functional equations. During the last decades several stability problems for various functional equations have been investigated by many mathematicians; we refer the reader to the monographs $[3,5,6,7,11,12,13,16,21,24]$.

The functional equation

$$
f(x+y)+f(x-y)=2 f(x)+2 f(y),
$$

is called the quadratic functional equation and every solution of the quadratic equation (1.1) is said to be a quadratic function. It is well known that a function $f$ between two real vector spaces is quadratic if and only if there exists a unique symmetric bi-additive function $B$ such that $f(x)=B(x, x)$ for all $x$, where

$$
B(x, y)=\frac{1}{4}(f(x+y)-f(x-y))
$$

(see [17]).

The Hyers-Ulam stability problem for the quadratic functional equation was solved by Skof [25] and, independently, by Cholewa [4]. An analogous result for quadratic stochastic processes was obtained by Nikodem [18]. In [2], Czerwik proved the generalized Hyers-Ulam stability of the quadratic functional equation. Jung [15] dealt with stability problems for the quadratic functional equation of Pexider type.

Jun and Kim [14] introduced the following functional equation

$$
f(2 x+y)+f(2 x-y)=2 f(x+y)+2 f(x-y)+12 f(x),
$$

and established the general solution and the generalized Hyers-Ulam-Rassias stability for functional equation (1.3). Obviously, the $f(x)=x^{3}$ satisfies functional equation (1.3), so it is natural to call (1.3) the cubic functional equation. Every solution of the cubic functional equation is said to be a cubic function. Jun and Kim proved also that a function $f$ between two real vector spaces $X$ and $Y$ is a solution of (1.3) if and only if there exists a unique function $C: X \times X \times X \longrightarrow Y$ such that $f(x)=C(x, x, x)$ for all $x \in X$, moreover, $C$ is symmetric for each 
fixed one variable and is additive for fixed two variables. Later a number of mathematicians worked on the stability of some types of the cubic equation [23].

Let $X, Y$ and $Z$ be vector spaces on $\mathbb{R}$ or $\mathbb{C}$. We say that a mapping $f$ : $X \times Y \rightarrow Z$ is additive-quadratic if $f$ satisfies the following system of functional equations:

$$
\left\{\begin{array}{l}
f\left(x_{1}+x_{2}, y\right)=f\left(x_{1}, y\right)+f\left(x_{2}, y\right) \\
f\left(x, y_{1}+y_{2}\right)+f\left(x, y_{1}-y_{2}\right)=2 f\left(x, y_{1}\right)+2 f\left(x, y_{2}\right)
\end{array}\right.
$$

for all $x, x_{1}, x_{2} \in X$ and $y, y_{1}, y_{2} \in Y$. See the following examples.

- Let $X=Y=Z=\mathbb{R}$. We define $f$ by $f(x, y)=x y^{2}$. It is easy to see that $f$ is an additive-quadratic map.

- Let $X$ be a normed space, and let $Y=Z=X$. Suppose $f(x, y)=x\|y\|^{2}$. Then $f$ is an additive-quadratic map.

- Let $A$ be an algebra, and let $X$ be a right $A$-module. Set $Z=X$ and $Y=A$. Suppose $f(x, a)=x a^{2}$. Then $f$ is an additive-quadratic map.

We recall some basic facts concerning $F$-spaces. In functional analysis, an $F$-space is a vector space $V$ over the real or complex numbers together with a metric $d: V \times V \rightarrow \mathbb{R}$ so that scalar multiplication in $V$ is continuous with respect to $d$ and the standard metric on $\mathbb{R}$ or $\mathbb{C}$. Addition in $V$ is continuous with respect to $d$. The metric is translation-invariant, i.e. $d(x+a, y+a)=d(x, y)$ for all $x, y$ and $a$ in $V$. The metric space $(V, d)$ is complete.

Some authors call these spaces "Frchet spaces", but usually the term Frchet space is reserved for locally convex $F$-spaces. The metric may or may not necessarily be part of the structure on an $F$-space; many authors only require that such a space be metrizable in a manner that satisfies the above properties. Trivially, every Banach space is a $F$-space as the norm induces a translation invariant metric and the space is complete with respect to this metric. The $L_{p}$ spaces are $F$-spaces for all $p>0$ and for $p=1$ they are locally convex and thus Frchet spaces and even Banach spaces. So for example, $L_{\frac{1}{2}}([0,1])$ is a $F$-space, which is not a Banach space.

\section{Main Results}

We start our work with the following result, which explain the relation between additive-quadratic maps and cubic maps.

Theorem 2.1. Let $X, Z$ be vector spaces. A mapping $g: X \rightarrow Z$ is cubic if and only if there exists an additive-quadratic mapping $f: X \times X \rightarrow Z$ such that $g(x)=f(x, x)$ and that

$$
f(y, 2 x+y)-f(y, 2 x-y)=2(f(y, x+y)-f(y, x-y))
$$

for all $x, y \in X$.

Proof. Let $g: X \rightarrow Z$ be a cubic mapping. Then there exists a mapping $C: X \times$ $X \times X \rightarrow Z$ such that $C$ is symmetric for each fixed one variable and is additive for fixed two variables. Define $f: X \times X \rightarrow Z$ by $f(x, y)=C(x, y, y)$. One can 
show that $f$ is additive quadratic mapping satisfies $f(y, 2 x+y)-f(y, 2 x-y)=$ $2(f(y, x+y)-f(y, x-y))$ for all $x, y \in X$. For the converse, let $f: X \times X \rightarrow Z$ be an additive-quadratic mapping such that $f(y, 2 x+y)-f(y, 2 x-y)=2(f(y, x+$ $y)-f(y, x-y))$ for all $x, y \in X$. Then it is easy to see that the mapping $g: X \rightarrow Z$, defined by $g(x)=f(x, x)$ for all $x \in X$, is cubic.

We now investigate the generalized Hyers-Ulam-Rassias stability problem for system of functional equations (1.4). From now on, let $X$ be a real vector space and $Y$ be a real $F$-space by metric $d$. Let $f: X \times X \rightarrow Y$ be a function then we define $\Delta_{f}, D_{f}: X \times X \times X \rightarrow \mathbb{R}$ by

$$
\begin{gathered}
D_{f}\left(x_{1}, x_{2}, y\right)=d\left(f\left(x_{1}+x_{2}, y\right), f\left(x_{1}, y\right)+f\left(x_{2}, y\right)\right) \\
\Delta_{f}\left(x, y_{1}, y_{2}\right)=d\left(f\left(x, y_{1}+y_{2}\right)+f\left(x, y_{1}-y_{2}\right), 2 f\left(x, y_{1}\right)+2 f\left(x, y_{2}\right)\right)
\end{gathered}
$$

for all $x, x_{1}, x_{2}, y, y_{1}, y_{2} \in X$.

Theorem 2.2. Let $\phi, \psi: X \times X \times X \rightarrow[0, \infty)$ be mappings satisfying

$$
\sum_{i=0}^{\infty} \frac{\psi\left(2^{i+1} x, 2^{i} y, 2^{i} y\right)+\phi\left(2^{i} x, 2^{i} x, 2^{i} y\right)}{8^{i}}<\infty
$$

for all $x, y \in X$, and

$$
\lim \frac{\psi\left(2^{n} x, 2^{n} y, 2^{n} z\right)+\phi\left(2^{n} x, 2^{n} y, 2^{n} z\right)}{8^{n}}=0
$$

for all $x, y, z \in X$. If $f: X \times X \rightarrow Y$ is a mapping such that

$$
\begin{gathered}
D_{f}\left(x_{1}, x_{2}, y\right) \leq \phi\left(x_{1}, x_{2}, y\right), \\
\Delta_{f}\left(x, y_{1}, y_{2}\right) \leq \psi\left(x, y_{1}, y_{2}\right)
\end{gathered}
$$

for all $x, y, x_{1}, x_{2}, y_{2}, y_{2} \in X$, then there exists a unique additive-quadratic mapping $T: X \times X \rightarrow Y$ satisfying (1.4) and

$$
d(f(x, y), T(x, y)) \leq \frac{1}{8} \sum_{i=0}^{\infty} \frac{\psi\left(2^{i+1} x, 2^{i} y, 2^{i} y\right)}{8^{i}}+\frac{1}{2} \sum_{i=0}^{\infty} \frac{\phi\left(2^{i} x, 2^{i} x, 2^{i} y\right)}{8^{i}}
$$

for all $x, y \in X$.

Proof. Putting $x_{1}=x_{2}=x$ in (2.1), we get

$$
d(f(2 x, y), 2 f(x, y)) \leq \phi(x, x, y) .
$$

Replacing $y_{1}, y_{2}$ by $y$ in (2.2) to obtain

$$
d(f(x, 2 y), 4 f(x, y)) \leq \psi(x, y, y) .
$$

Replacing $x$ by $2 x$ in (2.5), yields

$$
d(f(2 x, 2 y), 4 f(2 x, y)) \leq \psi(2 x, y, y) .
$$

Combining (2.4) and (2.6), we lead to

$$
d\left(\frac{1}{8} f(2 x, 2 y), f(x, y)\right) \leq \frac{1}{2} \psi(x, x, y)+\frac{1}{8} \psi(2 x, y, y) .
$$


From the inequality (2.7) we use iterative methods and induction on $n$ to prove our next relation:

$$
d\left(\frac{1}{8^{n}} f\left(2^{n} x, 2^{n} y\right), f(x, y)\right) \leq \frac{1}{2} \sum_{i=0}^{n-1} \psi\left(2^{n} x, 2^{n} x, 2^{n} y\right)+\frac{1}{8} \sum_{i=0}^{n-1} \frac{1}{8^{n}} \psi\left(2^{n+1} x, 2^{n} y, 2^{n} y\right) .
$$

We divide (2.8) by $8^{m}$ and replace $x$ by $2^{m} x$ to obtain that

$$
\begin{aligned}
d\left(\frac{1}{8^{m+n}}\right. & \left.f\left(2^{m+n} x, 2^{m+n} y\right), \frac{1}{8^{m}} f\left(2^{m} x, 2^{m} y\right)\right) \leq \frac{1}{2} \sum_{i=0}^{n-1} \psi\left(2^{m+n} x, 2^{m+n} x, 2^{m+n} y\right) \\
& +\frac{1}{8} \sum_{i=0}^{n-1} \frac{1}{8^{n+m}} \psi\left(2^{m+n+1} x, 2^{m+n} y, 2^{m+n} y\right) .
\end{aligned}
$$

This shows that $\left\{\frac{1}{8^{n}} f\left(2^{n} x, 2^{n} y\right)\right\}$ is a Cauchy sequence in $\mathrm{Y}$ by taking the limit $m \rightarrow \infty$. Since Y is a Banach space, it follows that the sequence $\left\{\frac{1}{8^{n}} f\left(2^{n} x, 2^{n} y\right)\right\}$ converges. We define $T: X \times X \rightarrow Y$ by $T(x, y)=\lim _{n \rightarrow \infty} \frac{1}{8^{n}} f\left(2^{n} x, 2^{n} y\right)$ for all $x, y \in X$. It follows from (2.1) that

$$
D_{T}\left(x_{1}, x_{2}, y\right)=\lim _{n \rightarrow \infty} \frac{1}{8^{n}} D_{f}\left(2^{n} x_{1}, 2^{n} x_{2}, 2^{n} y\right) \leq \lim _{n \rightarrow \infty} \frac{1}{8^{n}} \phi\left(2^{n} x_{1}, 2^{n} x_{2}, 2^{n} y\right)=0
$$

for all $x_{1}, x_{2}, y \in X$. Also it follows from (2.2) that

$$
\Delta_{T}\left(x, y_{1}, y_{2}\right)=\lim _{n \rightarrow \infty} \frac{1}{8^{n}} \Delta_{f}\left(2^{n} x, 2^{n} y_{1}, 2^{n} y_{2}\right) \leq \lim _{n \rightarrow \infty} \frac{1}{8^{n}} \phi\left(2^{n} x, 2^{n} y_{1}, 2^{n} y_{2}\right)=0
$$

for all $x, y_{1}, y_{2} \in X$. This means that $T$ is additive-quadratic. It remains to show that $T$ is unique. Suppose that there exists another additive-quadratic mapping $T^{\prime}: X \times X \rightarrow Y$ which satisfies (1.4) and (2.3). Since $\frac{1}{8^{n}} T\left(2^{n} x, 2^{n} y\right)=T(x, y)$, and $\frac{1}{8^{n}} T^{\prime}\left(2^{n} x, 2^{n} y\right)=T^{\prime}(x, y)$ for all $x, y \in X$, we conclude that

$$
\begin{aligned}
d\left(T(x, y), T^{\prime}(x, y)\right) & =\frac{1}{8^{n}} d\left(T\left(2^{n} x, 2^{n} y\right), T^{\prime}\left(2^{n} x, 2^{n} y\right)\right) \\
& \leq \frac{1}{8^{n}} d\left(T\left(2^{n} x, 2^{n} y\right), f\left(2^{n} x, 2^{n} y\right)\right)+d\left(f\left(2^{n} x, 2^{n} y\right), T^{\prime}\left(2^{n} x, 2^{n} y\right)\right) \\
& \leq 2\left[\frac{1}{8} \sum_{i=0}^{\infty} \frac{\psi\left(2^{n+i+1} x, 2^{n+i} y, 2^{n+i} y\right)}{8^{n+i}}+\frac{1}{2} \sum_{i=0}^{\infty} \frac{\phi\left(2^{n+i} x, 2^{n+i} x, 2^{n+i} y\right)}{8^{n+i}}\right]
\end{aligned}
$$

for all $x, y \in X$. By letting $n \rightarrow \infty$ in this inequality, it follows that $T(x, y)=$ $T^{\prime}(x, y)$ for all $x, y \in X$, which gives the conclusion.

Let $X, Y$ and $Z$ be vector spaces on $\mathbb{R}$ or $\mathbb{C}$. We say that a mapping $f$ : $X \times Y \rightarrow Z$ is quadratic-additive if $f$ satisfies

$$
\left\{\begin{array}{l}
f\left(x_{1}+x_{2}, y\right)+f\left(x_{1}-x_{2}, y\right)=2 f\left(x_{1}, y\right)+2 f\left(x_{2}, y\right), \\
f\left(x, y_{1}+y_{2}\right)=f\left(x, y_{1}\right)+f\left(x, y_{2}\right)
\end{array}\right.
$$

for all $x, x_{1}, x_{2} \in X$ and $y, y_{1}, y_{2} \in Y$. 
Theorem 2.3. Let $\phi, \psi: X \times X \times X \rightarrow[0, \infty)$ be mappings satisfying

$$
\sum_{i=0}^{\infty} \frac{\psi\left(2^{i} x, 2^{i} x, 2^{i+1} y\right)+\phi\left(2^{i} x, 2^{i} y, 2^{i} y\right)}{8^{i}}<\infty
$$

for all $x, y \in X$, and

$$
\lim \frac{\psi\left(2^{n} x, 2^{n} y, 2^{n} z\right)+\phi\left(2^{n} x, 2^{n} y, 2^{n} z\right)}{8^{n}}=0
$$

for all $x, y, z \in X$. If $f: X \times X \rightarrow Y$ is a mapping such that

$$
\begin{gathered}
d\left(f\left(x_{1}+x_{2}, y\right)+f\left(x_{1}-x_{2}, y\right), 2 f\left(x_{1}, y\right)+2 f\left(x_{2}, y\right)\right) \leq \psi\left(x_{1}, x_{2}, y\right), \\
d\left(f\left(x, y_{1}+y_{2}\right), f\left(x, y_{1}\right)+f\left(x, y_{2}\right)\right) \leq \phi\left(x, y_{1}, y_{2}\right)
\end{gathered}
$$

for all $x, y, x_{1}, x_{2}, y_{2}, y_{2} \in X$, then there exists a unique additive-quadratic mapping $T: X \times X \rightarrow Y$ satisfying (1.4) and

$$
d(f(x, y), T(x, y)) \leq \frac{1}{2} \sum_{i=0}^{\infty} \frac{\psi\left(2^{i} x, 2^{i} y, 2^{i} y\right)}{8^{i}}+\frac{1}{8} \sum_{i=0}^{\infty} \frac{\phi\left(2^{i} x, 2^{i} x, 2^{i+1} y\right)}{8^{i}}
$$

for all $x, y \in X$.

Proof. Put $f_{0}(x, y)=f(y, x), \phi_{0}(x, y, z)=\phi(z, y, x)$ and $\psi_{0}(x, y, z)=\psi(z, y, x)$, for all $x, y, z \in X$. Then by above theorem, there exists a unique additivequadratic mapping $T_{0}: X \times X \rightarrow Y$ satisfies

$$
d\left(f_{0}(x, y), T_{0}(x, y)\right) \leq \frac{1}{8} \sum_{i=0}^{\infty} \frac{\psi_{0}\left(2^{i+1} x, 2^{i} y, 2^{i} y\right)}{8^{i}}+\frac{1}{2} \sum_{i=0}^{\infty} \frac{\phi_{0}\left(2^{i} x, 2^{i} x, 2^{i} y\right)}{8^{i}}
$$

for all $x, y \in X$. Now, we put $T(x, y)=T_{0}(y, x)$ for all $x, y \in X$. Hence, $T$ is a unique quadratic-additive map satisfies (2.10).

Corollary 2.4. Let $X$ be a vector space and $Y$ be a Banach space. Suppose the mappings $\phi, \psi: X \times X \times X \rightarrow[0, \infty)$ satisfying

$$
\sum_{i=0}^{\infty} \frac{\psi\left(2^{i+1} x, 2^{i} y, 2^{i} y\right)+\phi\left(2^{i} x, 2^{i} x, 2^{i} y\right)}{8^{i}}<\infty
$$

for all $x, y \in X$, and

$$
\lim \frac{\psi\left(2^{n} x, 2^{n} y, 2^{n} z\right)+\phi\left(2^{n} x, 2^{n} y, 2^{n} z\right)}{8^{n}}=0
$$

for all $x, y, z \in X$. If $f: X \times X \rightarrow Y$ is a mapping such that

$$
\begin{gathered}
\left\|f\left(x_{1}+x_{2}, y\right)-f\left(x_{1}, y\right)+f\left(x_{2}, y\right)\right\| \leq \phi\left(x_{1}, x_{2}, y\right), \\
\left\|f\left(x, y_{1}+y_{2}\right)+f\left(x, y_{1}-y_{2}\right)-2 f\left(x, y_{1}\right)-2 f\left(x, y_{2}\right)\right\| \leq \psi\left(x, y_{1}, y_{2}\right)
\end{gathered}
$$

for all $x, y, x_{1}, x_{2}, y_{2}, y_{2} \in X$, then there exists a unique additive-quadratic mapping $T: X \times X \rightarrow Y$ satisfying (1.4) and

$$
\|f(x, y)-T(x, y)\| \leq \frac{1}{8} \sum_{i=0}^{\infty} \frac{\psi\left(2^{i+1} x, 2^{i} y, 2^{i} y\right)}{8^{i}}+\frac{1}{2} \sum_{i=0}^{\infty} \frac{\phi\left(2^{i} x, 2^{i} x, 2^{i} y\right)}{8^{i}}
$$


for all $x, y \in X$.

Proof. It follows from theorem 2.2. by Putting $d(a, b)=\|a-b\|$ for all $a, b \in$ $Y$.

We are going to investigate the Hyers-Ulam -Rassias stability problem for system of functional equations (1.4).

Corollary 2.5. Let $\mathrm{\epsilon}>0, p<3$, and let $X, Y$ be a normed space a Banach space, respectively. If $f: X \times X \rightarrow Y$ is a mapping such that

$$
\begin{aligned}
\operatorname{Max}\{ & \left\|f\left(x, y_{1}+y_{2}\right)+f\left(x, y_{1}-y_{2}\right)-2 f\left(x, y_{1}\right)-2 f\left(x, y_{2}\right)\right\| \\
& \left.,\left\|f\left(x_{1}+x_{2}, y\right)-f\left(x_{1}, y\right)+f\left(x_{2}, y\right)\right\|\right\} \\
& \leq \epsilon\left(\operatorname{Min}\left\{\left\|x_{1}\right\|^{p}+\left\|x_{2}\right\|^{p}+\|y\|^{p},\|x\|^{p}+\left\|y_{1}\right\|^{p}+\left\|y_{2}\right\|^{p}\right\}\right)
\end{aligned}
$$

for all $x, y, x_{1}, x_{2}, y_{2}, y_{2} \in X$, then there exists a unique additive-quadratic mapping $T: X \times X \rightarrow Y$ satisfying (1.4) and

$$
\|f(x, y)-T(x, y)\| \leq \frac{\epsilon}{8-2^{p}}\left(\left(2^{p}+9\right)\|x\|^{p}+5\|y\|^{p}\right)
$$

for all $x, y \in X$.

Proof. It follows from corollary 2.4. by Putting $\phi(a, b, c)=\psi(a, b, c)=\|a\|^{p}+$ $\|b\|^{p}+\|c\|^{p}$ for all $a, b, c \in X$.

By Corollary 2.5, we solve the following Hyers-Ulam stability problem for system of functional equations (1.4).

Corollary 2.6. Let $\epsilon>0$, and let $X, Y$ be a normed space a Banach space, respectively. If $f: X \times X \rightarrow Y$ is a mapping such that

$$
\begin{aligned}
& \operatorname{Max}\left\{\left\|f\left(x, y_{1}+y_{2}\right)+f\left(x, y_{1}-y_{2}\right)-2 f\left(x, y_{1}\right)-2 f\left(x, y_{2}\right)\right\|\right. \\
& \left.\quad,\left\|f\left(x_{1}+x_{2}, y\right)-f\left(x_{1}, y\right)+f\left(x_{2}, y\right)\right\|\right\} \\
& \quad \leq \epsilon
\end{aligned}
$$

for all $x, y, x_{1}, x_{2}, y_{2}, y_{2} \in X$, then there exists a unique additive-quadratic mapping $T: X \times X \rightarrow Y$ satisfying (1.4) and

$$
\|f(x, y)-T(x, y)\| \leq \frac{15 \epsilon}{7}
$$

for all $x, y \in X$.

Similarly we can prove the Hyers-Ulam-Rassias stability and the Hyers-Ulam stability problems for quadratic-additive maps. 


\section{REFERENCES}

1. T. Aoki, On the stability of the linear transformationin Banach spaces, J. Math. Soc. Japan 2 (1950) 64-66.

2. S. Czerwik, On the stability of the quadratic mapping in normed spaces, Abh. Math. Sem. Univ. Hamburg 62 (1992), 59-64.

3. S. Czerwik, Functional equations and inequalities in several variables, World Scientific, New Jersey, London, Singapore, Hong Kong, 2002. 1

4. P.W. Cholewa, Remarks on the stability of functional equations, Aequationes Math. 27 (1984), 76-86. 1

5. M. Eshaghi Gordji, A. Ebadian and S. Zolfaghari, Stability of a functional equation deriving from cubic and quartic functions, Abstract and Applied Analysis Volume 2008, Article ID 801904, 17 pages. 1

6. M. Eshaghi Gordji and H. Khodaei, Solution and stability of generalized mixed type cubic, quadratic and additive functional equation in quasi-Banach spaces, To appear in Nonlinear Analysis TMA. 1

7. M. Eshaghi,M. S. Moslehian, S. Kaboli and S. Zolfaghari, Stability of a mixed type additive, quadratic, cubic and quartic functional equation, To appear. 1

8. Z. Gajda, On stability of additive mappings, Internat. J. Math. Math. Sci. 14 (1991), 431434. 1

9. P. Guavruta, A generalization of the Hyers-Ulam-Rassias stability of approximately additive mappings, J. Math. Anal. Appl. 184 (1994), 431-436. 1

10. D.H. Hyers, On the stability of the linear functional equation, Proc. Nat. Acad. Sci. U.S.A. 27 (1941), 222-224. 1

11. D.H. Hyers, G. Isac and Th.M. Rassias, Stability of Functional Equations in Several Variables, Birkhauser, Basel, 1998. 1

12. G. Isac and Th. M. Rassias, On the Hyers-Ulam stability of $\psi$-additive mappings, J. Approx. Theory 72 (1993), 131-137.

13. G. Isac and Th.M. Rassias, Functional inequalities for approximately additive mappings, Stability of mappings of Hyers-Ulam type, 117-125, Hadronic Press Collect. Orig. Artic., Hadronic Press, Palm Harbor, FL, 1994. 1

14. K.W. Jun and H.M. Kim, The generalized Hyers-Ulam-Rassias stability of a cubic functional equation, J. Math. Anal. Appl. 274 (2002), 267-278. 1

15. S.-M. Jung,Stability of the quadratic equation of Pexider type, Abh. Math. Sem. Univ. Hamburg 70 (2000), 175-190. 1 1

16. S.-M. Jung, Hyers-Ulam-Rassias Stability of Functional Equations in Mathematical Analysis, Hadronic Press, Palm Harbor, Florida, 2001. 1 1

17. Pl. Kannappan, Quadratic functional equation and inner product spaces, Results Math. 27 (1995), 368-372. 1

18. K. Nikodem, On some properties of quadratic stochastic processes, Annales Math. Silesianae, 3 (1990), 59-69. 1

19. Th.M. Rassias, On the stability of the linear mapping in Banach spaces, Proc. Amer. Math. Soc., 72 (1978), 297-300. 1

20. Th.M. Rassias, Problem 16, 2, Report of the 27th International Symp.on Functional Equations, Aequationes Math. 39 (1990), 292-293. 1

21. Th.M. Rassias (ed.), Functional equations, inequalities and applications, Kluwer Academic Publishers, Dordrecht, Boston and London, 2003. 1

22. Th.M. Rassias and P. Semrl, On the behaviour of mappings which do not satisfy HyersUlam stability, Proc. Amer. Math. Soc., 114 (1992), 989-993. 1

23. P.K. Sahoo, A generalized cubic functional equation, Acta Math. Sin. (Engl. Ser.) 21 (2005), 1159-1166. 1 
24. S. Shakeri, Intuitionistic fuzzy stability of Jensen type mapping, 2 (2009), 105-112. 1

25. F. Skof, Proprietalocali e approssimazione di operatori, Rend. Sem. Mat. Fis. Milano 53 (1983), 113-129. 1

26. S.M. Ulam, Problems in Modern Mathematics, Chapter VI, Science Editions, Wiley, New York, 1964.

Department of Mathematics, Semnan University, P. O. Box 35195-363, Semnan, IRAN.

E-mail address: madjid.eshaghi@gmail.com 